\title{
Editorial
}

\section{New British recommendations for prevention of coronary heart disease in clinical practice}

Agreement between the European societies of atherosclerosis, cardiology, and hypertension on how to prevent coronary heart disease in clinical practice resulted in recommendations published in 1994. They were recently revised as a result of new evidence, particularly from clinical trials, of lowering of serum cholesterol. ${ }^{1}$ Underlying the concept of joint European recommendations has been the explicitly stated premise that they should be adapted to national conditions and resources.

With remarkable alacrity this has now been accomplished in the UK with the publication of recommendations from the British Cardiac Society, the British Hyperlipidaemia Association, and the British Hypertension Society (endorsed by the British Diabetic Association). ${ }^{2}$ As indicated in the title, the joint British recommendations on prevention of coronary heart disease in clinical practice closely mirror the European guidelines. One example is the simple recommendation that total cholesterol $<5 \mathrm{mmol} / 1$ $(190 \mathrm{mg} / \mathrm{dl})$ and low density lipoprotein (LDL) cholesterol $<3 \mathrm{mmol} / \mathrm{l}(115 \mathrm{mg} / \mathrm{dl})$ should be goals of treatment in both primary and secondary prevention.

The differences between the European and the British recommendations reflect intelligent adaptations to national conditions and concerns. The British as well as the European recommendations concerning primary prevention are based on an assessment of risk, in absolute terms, of developing clinical coronary disease within the next 10 years. This assessment is derived from the Framingham risk function incorporating age, sex, smoking status, systolic blood pressure, and a lipid measurement. The British recommendations have exploited the fact that, on a national level, it is possible to use the ratio of total to high density lipoprotein (HDL) cholesterol rather than total cholesterol as the lipid component of risk assessment. Far from new, this adaptation provides a more accurate assessment of the component of risk from dyslipidaemia.

An equally important difference derives from a mainly British debate concerning the level of risk at which to intervene in primary prevention, particularly with lipid lowering drugs. The European societies recommend 10 year risk of coronary heart disease of $20 \%$; the British societies recommend a staged approach. As a minimum, people at highest risk-defined as $>30 \%$ over the next 10 years - should be identified. Once they have been managed effectively, primary prevention with drugs should be progressively extended to those at lower levels of risk, down to a $15 \% 10$ year risk. Starting from a more conservative position, the British recommendations ultimately go one step further than the European ones.

The British recommendations concerning blood pressure are more complicated than the European ones, because they address certain problems in more detail. With the exception of guidelines developed in New Zealand, management of hypertension is based on blood pressure alone rather than on blood pressure in the context of absolute cardiovascular risk, including the risk of stroke. The British recommendations specifically state that blood pressure $>160 / 100 \mathrm{~mm} \mathrm{Hg}$ should be lowered irrespective of other factors because of established benefit in reducing the cerebrovascular component of overall cardiovascular risk. The latter should, moreover, be taken to be higher than the risk of coronary heart disease only. For example, a $15 \%$ risk of coronary heart disease corresponds to a $20 \%$ overall cardiovascular risk. In persons with blood pressure 140-160/90-100 $\mathrm{mm} \mathrm{Hg}$, the British societies recommend starting treatment when risk of coronary heart disease exceeds $15 \%$. As the threshold for lipid lowering drugs is currently $30 \%$, antihypertensive drugs will continue to be used more frequently than drugs to lower lipids. Priorities seem reversed here, because the main goal of the recommendations is prevention of coronary heart disease, a goal more easily reached by lowering lipids than lowering blood pressure.

After a myocardial infarction, and taking into consideration the resulting transitory lowering of cholesterol, how long should diet be given a chance to reduce cholesterol before starting a lipid lowering drug? The British societies propose a workable and sensible solution. If total cholesterol is $>6 \mathrm{mmol} / \mathrm{l}$ at admission, pharmacological as well as dietary treatment should be started before discharge, because only a small proportion of these patients will achieve cholesterol $<5 \mathrm{mmol} / 1$ and $\mathrm{LDL}<3 \mathrm{mmol} / \mathrm{l}$ with diet alone. Other patients can be followed on diet only, and if cholesterol exceeds $5 \mathrm{mmol} / \mathrm{l}$ at six weeks or later, they should also take a lipid lowering drug.

Recommendations must periodically be revised, and the present European and British recommendations will quickly become dated. Identification of new risk factors and results of clinical trials planned or in progress are soon likely to make further changes necessary. The best way to assess risk may also change. The coronary and other arteries can be imaged by ultrasonography, various form of computed tomography, and by nuclear magnetic resonance. Will measurements of degrees of atherosclerosis by such techniques supplement or even replace assessment of risk based on sex, age, cholesterol, blood pressure? These questions and others necessitate continued reappraisal of ways to prevent cardiovascular disease.

As they now appear, the British recommendations are practical and well argued. At the national level they reflect the European commitment to consensus between major scientific societies on how best to prevent coronary and other cardiovascular disease.

Department of Medicine and Cardiology,

OLE FAERGEMAN

Arhus Amtssygehus University Hospital,

Tage Hansens Gade 2,

8000 Arhus $C$, Denmark

email:ole.faergeman@aas.auh.dk

1 Wood D, De Backer G, Faergeman O, et al. Prevention of coronary heart disease in clinical practice: recommendations of the second joint task force of the joint European societies on coronary prevention. Eur Heart $\mathcal{F}$ 1998;19:1434-503.

2 British Cardiac Society, British Hyperlipidaemia Association, British Hypertension Society [endorsed by the British Diabetic Association]. Joint British recommendations on the prevention of coronary heart disease in

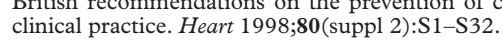

